\title{
Automatic Image Registration using 2D-Discrete Wavelet Transform
}

\author{
M. Deepa ${ }^{1 *}$ and T. Saravanan ${ }^{2}$ \\ 'Bharath University, Chennai -600073, Tamil Nadu, India; deepakirupa79@gmail.com \\ 2Department of ETC, Bharath University, Chennai - 600073, Tamil Nadu, India
}

\section{Abstract}

The main objective of the paper is automatic image registration using discrete wavelet transform. In this paper to register the images I use the discrete wavelet transform. The characteristic of the approach is that the image is sub divided in to four components that as Less-Less, Less-High, High-Less and High-High from that we took Less-Less for the further processing. The DWT is applied for both the reference and sensed image to extract the LL components and the feature power points are extracted using scale invariant feature transform to match the two images. The simulation results done using MATLAB.

Keywords: Discrete Wavelet Transform, Image Registration, Rotation, SIFT, Scaling, Translation

\section{Presentation}

In this paper, we propose and comprehend a novel picture selection framework to enroll picture with gigantic turn, scaling and interpretation. In an extensive variety of picture enrollment, Robustness of the estimation is the essential and required target. In any case, because of broadening of pictures obtained their substance and reason for their arrangement, it is verging on difficult to outline widespread strategy for picture enrollment that satisfy all prerequisites and suits a wide range of utilizations. A significant number of the picture enrollment procedures have been proposed and looked into. Picture enrollment procedures can be for the most part grouped in two classifications. Force based and include based. The primary class uses picture force to evaluate the parameters of a change between two pictures utilizing a methodology including all pixels of the picture. In second grouping a course of action of highlight centers expelled from a photo and uses only these isolated component concentrates rather than all entire picture pixels to acquire the change parameters. In this paper, estimation for highlight based picture enrollment is proposed. The proposed calculation depends on upon three focal steps, highlight point extraction, correspondence between disengaged part focuses and change parameter estimation.

\section{Discrete Wavelet Transform}

The Discrete Wavelet Transform (DWT), which relies on upon sub-band coding, is found to yield a snappy estimation of Wavelet Transform. It is undeniably not hard to execute and diminishes the number time and resources required. The two-dimensional DWT of a photo limit $\mathrm{s}(\mathrm{n} 1, \mathrm{n} 2)$ of size N1 x N2 might be communicated as

$$
\begin{aligned}
& W_{\phi}\left(j_{0}, k_{1}, k_{2}\right)=\frac{1}{\sqrt{N_{1} N_{2}}} \sum_{n_{1}=0}^{N_{1}-1} \sum_{n_{2}=0}^{N_{2}-1} s\left(n_{1}, n_{2}\right) \phi_{j_{0}, k_{1}, k_{2}}\left(n_{1}, n_{2}\right) \\
& W_{\psi}^{i}\left(j_{0}, k_{1}, k_{2}\right)=\frac{1}{\sqrt{N_{1} N_{2}}} \sum_{n_{1}=0}^{N_{1}-1} \sum_{n_{2}=0}^{N_{2}-1} s\left(n_{1}, n_{2}\right) \psi_{j_{0}, k_{1}, k_{2}}^{i}\left(n_{1}, n_{2}\right)
\end{aligned}
$$

where $\mathrm{i}=\{\mathrm{H}, \mathrm{V}, \mathrm{D}\}$ show the heading record of the wavelet limit. As in one-dimensional case j0 speaks to any beginning scale, which might be dealt with as $\mathrm{j} 0=0$. Given the above two experimental verbalizations are two-dimensional DWT. The stream diagram of the Discrete wavelet change sub band coding on the electronic picture is appeared in Figure 2, here $\mathrm{L}$ intimates low rehash bit, $\mathrm{H}$ recommends high rehash and the number 1 and 2 suggest the weakening level of the Discrete wavelet change. The aftereffect of the 2-D Discrete Wavelet Transform from level one to

${ }^{*}$ Author for correspondence 


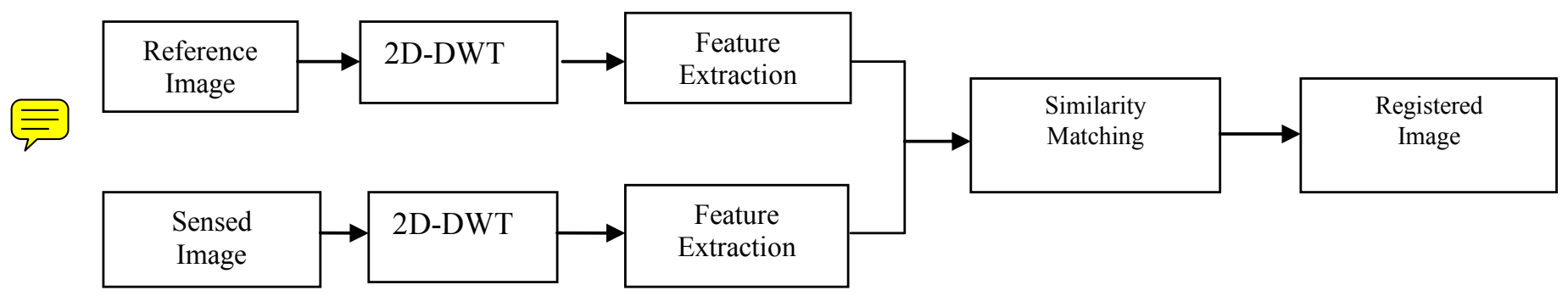

Figure 1. Block Diagram of Image Registration

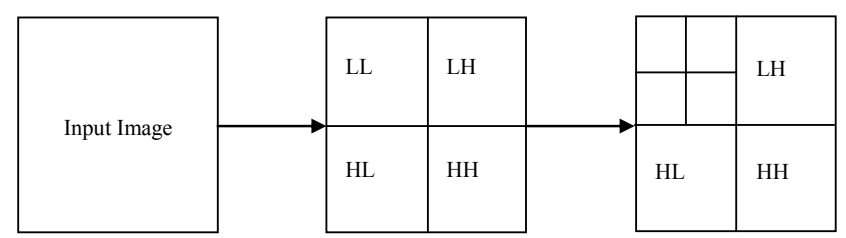

Figure 2. Flow chart of the DWT sub band coding

level three is appeared in Figure 3. The sub picture LL is the low rehash bit, it is the determined sub photograph of the essential picture; the sub picture HL is the part of the low rehash in bearing and the high rehash in vertical heading, it exhibits the level edge of the important picture; the sub picture LH is the piece of the high rehash in level course and the low rehash in vertical course, it shows the vertical edge of the principle picture; the sub picture $\mathrm{HH}$ is the high rehash area, it exhibits the slanted edge of the essential picture. It is displayed that most essentialness of the important picture is contained in the LL2 low rehash range. In addition, the other locale in the same size reflect edge highlight of the photograph in various core interests. Here we utilize the 2-D Discrete Haar wavelet change for the disintegrating of the photographs.

\section{Highlight Extraction}

Proposed calculation depends on highlight based picture enrollment. Basic errand we need to do is highlight extraction utilizing Discrete Wavelet Transforms. For the photo selection point of view, the separated element indicates must have satisfy some essential destinations like they ought to be sufficiently strong to some level of assortments in scale and the component ought to be able to be changed to adjust picture structures at various scales and the removed components ought to have a very much restricted sponsorship in the photo.

Approximated Features from both pictures are evacuated utilizing Discrete Wavelet Transform and Power

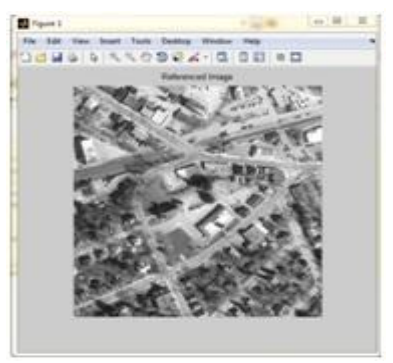

(a)

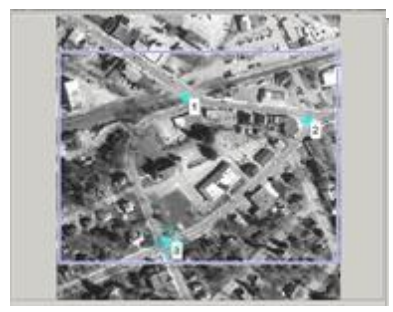

(c)

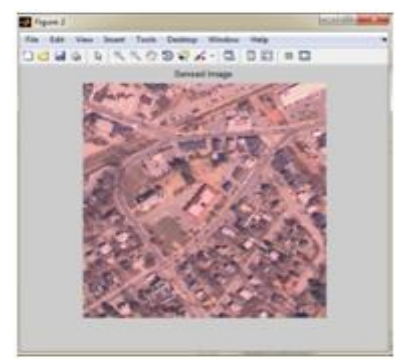

(b)

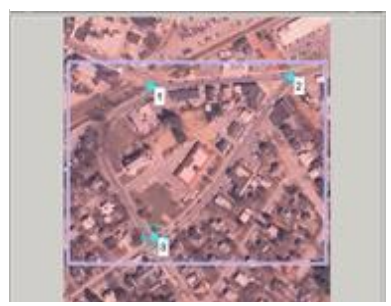

(d)
Figure 3. (a) Reference Image. (b) Reference Image Feature points. (c) Sensed Image. (d) Sensed Image Feature points

point correspondence and Feature arranging is master utilizing Scale-Invariant Feature Transform (SIFT). The scale invariant segment change (SIFT) calculation for picture highlights time which are invariant to picture understanding, scaling, change and generally invariant to light changes and relative projection.

In wake of removing the repeat part from the Discrete Wavelet Transform, making after strides are associated with perceive the segments and match the contrasting highlights from recognized and referenced picture. The accompanying system is selecting the force centers using the force determination gadget that gages the match of the power focuses taking into account the geometric relationship of the already chose power focuses.

\section{Similitude Coordinating}

All the past steps, for example, separating approximated level coefficients, for both reference and identified 
Images. In a matter of seconds, we have parts of Image 1 and segments of Image 2. Next step is to encourage the parts of both pictures. By utilizing the match Features limit, we will get Index Pairs, containing $\mathrm{P}$ sets of reports. These records contain highlights well while in travel to relate between the two information highlight sets. Next step is to recover the districts of taking a gander at communities for every photograph from the picked up Index Pairs. Our proposed Algorithm utilizes Nearest Neighbor Ratio technique for finding certifiable and false matches. By then the photographs will be selected ordinarily.

\section{Test Result}

The analysis result is appeared underneath Figure 3(a) demonstrates the reference picture Figure 3(b) demonstrates the element power focuses Figure 3(c) demonstrates the detected picture Figure 3(d) demonstrates the power point for detected pictures these power focuses are coordinated utilizing change invariance highlight change in the event that it coordinates the pictures are enlisted.

\section{Conclusion}

In this paper, another element based picture enlistment methodology is proposed. This kind of methodology gives another measurement to the current component based picture enlistment techniques. After effective element extraction utilizing Discrete Wavelet, correspondence between extricated highlight focuses is set up utilizing Scale Invariant Method. With this proposed count, we picked pictures with any level of turn and scaling.

\section{References}

1. Hong G, Zhang Y. Combination of feature-based and area based image registration technique for high resolution remote sensing image. Geoscience and Remote Sensing Symposium. IGARSS: Barcelona; 2007.

2. Shi H, Luo S. Image registration using the shift-insensitive discrete wavelet transform. Proceedings of the IEEE International Conference on Medical Image Analysis and Clinical Applications (MIACA). Guangdong; 2010. p. 46-49.

3. Sarvaiya JN, Patnaik S. Automatic image registration using mexican hat wavelet, invariant moment, and radon transform. International Journal of Advanced Computer Science and Applications. 2011; Special Issue on Image Processing and Analysis: 75-84.

4. Rajaei A. Feature extraction of currency notes: an approach based on wavelet transform. Proceedings of the IEEE International Conference on Advanced Computing \& Communication Technologies (ACCT). 2012: 255-58.

5. Wang C, Gao RX. Wavelet transform with spectral post-processing for enhanced feature extraction. IEEE Instrumentation and Measurement Technology Conference. 2003; 52(4):296-1301.

6. Quddus A, Basir O. Wavelet-based medical image registration for retrieval applications. Proceedings of the 2008 International Conference on Bio-Medical Engineering and Informatics. 2008; 02:301-5.

7. Yasein MS, Agathoklis P. A robust, feature-based algorithm for aerial image registration. Proceedings of the IEEE International Symposium on Industrial Electronics (ISIE 2007); Vigo: Spain; 2007. p. 1731-36.

8. Zitova B, Flusser J. Image registration methods: a survey. Image and Vision Computing. 2003; 21(11):977-1000.

9. Shahadi HI, Jidin R, Way WH. Lossless audio steganography based on lifting wavelet transform and dynamic stego key. Indian Journal of Science and Technology. 2014 Mar; 7(3):323-34. DOI: 10.17485/ijst/2014/v7i3/47652. 